\title{
Adaptive Meshless Centres and RBF Stencils for Poisson Equation
}

\author{
Oleg Davydov* and Dang Thi Oanh ${ }^{\dagger \ddagger}$
}

December 24, 2009

\begin{abstract}
We consider adaptive meshless discretisation of the Dirichlet problem for Poisson equation based on numerical differentiation stencils obtained with the help of radial basis functions. New meshless stencil selection and adaptive refinement algorithms are proposed in 2D. Numerical experiments show that the accuracy of the solution is comparable with, and often better than that achieved by the mesh-based adaptive finite element method.
\end{abstract}

\section{Introduction}

Motivated by the difficulties to create, maintain and update complex meshes needed for the standard finite difference, finite element or finite volume discretisations of the partial differential equations, meshless methods have become a subject of intensive research, see e.g. $[15,30,33]$ and references therein.

Even though the most attention has been paid to the methods based on the discretisation of the PDE in the weak form, the strong form methods such as collocation or generalised finite differences remain an attractive alternative as they avoid costly numerical integration of the non-polynomial shape functions on non-standard domains often encountered in the weak form methods.

It is a common idea to construct the shape functions such that their linear combinations reproduce polynomials of certain degree, or to generate finite difference stencils from numerical differentiation formulas obtained by imposing the conditions of polynomial exactness, thus exploiting the local approximation power of polynomials. This is not necessary in the approaches based on radial basis functions (RBFs). RBF approximation methods $[5,15,43]$ have gained popularity as a tool for meshless scattered data

*Department of Mathematics, University of Strathclyde, 26 Richmond Street, Glasgow G1 1XH, Scotland, oleg.davydov@strath.ac.uk

${ }^{\dagger}$ Department of Computer Science, Faculty of Information Technology - Thai Nguyen University, Quyet Thang Commune, Thai Nguyen City, Viet Nam, dtoanhtn@gmail.com

${ }^{\ddagger}$ The second author was supported in part by the National Foundation for Science and Technology Development (NAFOSTED) and a Natural Science Research Project of the Ministry of Education and Training. 
modelling. Because theoretical error bounds predict the spectral convergence of RBF interpolants when the density of interpolation points increases indefinitely, the most popular applications of RBF to solving partial differential equations are via global collocation or pseudospectral methods that can achieve spectral convergence orders, see [15]. Local weak form methods relying on shape functions resulting from RBF interpolation have been proposed e.g. in $[25,26]$.

We are interested in adaptive discretisation techniques for the Poisson equation based on generalised finite difference stencils generated with the help of RBF interpolation studied recently in $[21,41,42,44,4]$. Note that the best known approach to the generation of finite difference stencils on irregular centres is the polynomial least squares method $[19,23,38,3]$. This and related methods are often understood as local collocation. The local shape functions are not necessarily polynomials, they can be generated by moving least squares for example, but typically they are nevertheless guaranteed to reproduce polynomials of certain degree $[24,34,20,32,35]$. Local numerical differentiation with RBF rather than polynomials has been explored for solving certain time dependent PDEs in $[18,6]$.

Our interest to RBF numerical differentiation stencils stems from our experience with localised scattered data fitting methods, where RBF approximation has proved to be more robust on highly irregular data than local polynomial least squares $[9,10,8]$. Whereas the local polynomial approximations require careful adaptive degree selection because the minimum singular values of the least squares matrices directly influence the approximation error $[7,11], \mathrm{RBF}$ approximations are usually satisfactory as soon as the local RBF centres are sufficiently uniformly distributed, see also [16].

In this paper we address two main problems which have not received much attention in the literature. The first problem is the selection of RBF stencil supports on adaptively distributed centres. Indeed, recall that in the weak form meshless methods the stencil supports (the sets of centres with nonzero entries of the stiffness matrix in a given row) are determined by the domains of influence of the centres. To obtain the entries themselves, integration over intersections of the domains of influence is needed, which requires accurate quadrature formulas and may be difficult if the domains of influence are complicated. In contrast to this, generalised finite difference methods allow a lot of flexibility in the choice of stencil supports. For the polynomial methods, sophisticated algorithms have been developed, see [38, 20, 35]. We are not aware, however, of any work in this direction for the RBF based generalised finite differences. In Section 5 we propose an algorithm (Algorithm 1) for the selection of stencil supports that works successfully in our numerical tests on adaptive centres in 2D, in particular on the centres generated by a finite element method. To ensure a fair comparison it is important that the matrices of the sparse linear systems that arise from both methods have a comparable number of nonzero entries. This requirement dictates, in particular, the shape and the size of the RBF stencils consisting of a central point and 6 or 5 nearby points.

The second problem is that of meshless adaptive refinement of the discretisation. Clearly, one of the key ingredients of a successful numerical method for PDEs is the availability of algorithms for (nearly) optimal spatial distribution of the degrees of freedom, which is solved by the mesh generation techniques in the mesh-based methods, and needs to be addressed without using meshes in the case of meshless methods. If the 
locations of the singularities of the solution are known a priori, then meshless methods for the generation of well-distributed centres with respect to a given density distribution (see e.g. $[22,13,28]$ ) can be used. Otherwise, the ideas from the adaptive finite element method [2] can be adopted. Recall that the main ingredients of an adaptive method are an error indicator and a refinement algorithm. Adaptive methods have been intensively studied for the weak form meshless methods (see [14, 27, 36] and further references in [33, Section 2.10]), leading in particular to the error indicators based on a posteriori error estimates similar to those used in the highly successful adaptive finite element methods. Papers $[3,35]$ are devoted to the adaptive algorithms for the generalised finite differences and related polynomial-based methods. In Section 6 we present an algorithm (Algorithm 2) for adaptive meshless refinement of the set of centres which produces well distributed centres in our numerical tests and, together with Algorithm 1 leads to a purely meshless adaptive numerical method for the Poisson equation on domains in 2D that can compete with the adaptive finite element method, see the numerical experiments in Section 6.

The paper is organised as follows. In Section 2 we discuss a general form discretisation of the Dirichlet problem for the Poisson equation which covers finite differences, finite elements (when the use of numerical quadrature is taken into account) and the collocation type meshless methods. Section 3 describes the computation of stencil coefficients for three types of RBF stencils considered in this paper. In Section 4 we provide a numerical experiment where on a series of triangulations generated by an adaptive finite element algorithm, the standard FEM stencil coefficients are replaced by RBF coefficients. The performance of the RBF methods in this setting seems inferior. Sections 5 and 6 are devoted to our main topics, meshless stencil support selection and adaptive meshless refinement, respectively. Finally, Section 7 provides a short conclusion and a discussion of future work.

\section{Discretisation of Poisson equation}

Consider the Dirichlet problem for the Poisson equation in a bounded domain $\Omega \subset \mathbb{R}^{d}$ : given a function $f$ defined on $\Omega$, and a function $g$ defined on $\partial \Omega$ find $u$ such that

$$
\Delta u=f \text { on } \Omega ;\left.\quad u\right|_{\partial \Omega}=g .
$$

This problem can be discretised with the help of two finite sets $\Xi \subset \bar{\Omega}$ (discretisation centres) and $\Theta \subset \Omega$ (collocation centres) as follows. For each $\zeta \in \Xi \backslash \partial \Xi$, where $\partial \Xi:=\Xi \cap \partial \Omega$, choose two sets $\Xi_{\zeta} \subset \Xi$ and $\Theta_{\zeta} \subset \Theta$, as well as the weights $w_{\zeta, \xi} \in \mathbb{R}$, $\xi \in \Xi_{\zeta}$, and $\sigma_{\zeta, \theta} \in \mathbb{R}, \theta \in \Theta_{\zeta}$. We assume that each $\Xi_{\zeta}$ contains $\zeta$. A discretisation of the Dirichlet problem (1) is given by the following linear system with respect to the vector $\hat{u}=\left(\hat{u}_{\xi}\right)_{\xi \in \Xi}$

$$
\sum_{\xi \in \Xi_{\zeta}} w_{\zeta, \xi} \hat{u}_{\xi}=\sum_{\theta \in \Theta_{\zeta}} \sigma_{\zeta, \theta} f(\theta), \quad \zeta \in \Xi \backslash \partial \Xi ; \quad \hat{u}_{\xi}=g(\xi), \quad \xi \in \partial \Xi .
$$

If (2) is nonsingular, then its solution $\hat{u}$ can be compared with the vector $u_{\mid \Xi}=(u(\xi))_{\xi \in \Xi}$ of the discretised exact solution of (1). 
Many existing numerical methods for Dirichlet problem rely on solving a linear system in the form (2). To define a particular method, one needs to describe 1) a centre generation algorithm, that is a method to generate $\Xi$ and $\Theta$, and 2) a stencil selection algorithm, a method to choose subsets $\Xi_{\zeta}, \Theta_{\zeta}$ and coefficients $\left(w_{\zeta, \xi}\right)_{\xi \in \Xi_{\zeta}},\left(\sigma_{\zeta, \theta}\right)_{\theta \in \Theta_{\zeta}}$ for each $\zeta \in \Xi \backslash \partial \Xi$. We will often refer to the set $\Xi_{\zeta}$ as stencil support.

A standard finite difference method on a square domain $\Omega \subset \mathbb{R}^{2}$ is obtained if the elements of $\Xi$ and $\Theta$ are taken to be the nodes of a uniform grid of size $h$, and the classical 5-point stencil is given by $\Theta_{\zeta}=\{\zeta\}, \sigma_{\zeta, \zeta}=1, \Xi_{\zeta}=\{\zeta, \zeta \pm(h, 0), \zeta \pm(0, h)\}$, $w_{\zeta, \zeta}=-4 / h^{2}$ and $w_{\zeta, \xi}=1 / h^{2}, \xi \in \Xi_{\zeta} \backslash\{\zeta\}$. Further versions of the finite difference method derive the stencils from appropriate numerical differentiation formulas for the Laplace operator,

$$
\Delta u(\zeta) \approx \sum_{\xi \in \Xi_{\zeta}} w_{\zeta, \xi} u(\xi)
$$

The finite element method also leads to a system in the form (2) when the element load vectors are computed by a numerical quadrature. For example, in the linear triangle finite element method with midpoint quadrature, the centres in $\Xi$ are the vertices of a triangle mesh, $\Theta$ consists of the barycentres of the triangles, and the stencils are selected as follows: for each $\zeta \in \Xi \backslash \partial \Xi$, the set $\Theta_{\zeta}$ consists of the barycentres $\theta$ of the triangles $T_{\theta}$ attached to $\zeta, \Xi_{\zeta}$ is the set of the vertices of the same triangles $T_{\theta}, \theta \in \Theta_{\zeta}$, and the coefficients are given by $\sigma_{\zeta, \theta}:=\operatorname{area}\left(T_{\theta}\right) / 3, w_{\zeta, \xi}:=-\int_{\Omega} \nabla \phi_{\xi} \nabla \phi_{\zeta}, \xi \in \Xi_{\zeta}$, where $\phi_{\xi}$ denotes the hat function centred at $\xi$.

Recall that the role of a mesh in a mesh-based method is three-fold: It discretises the domain $\Omega$, provides the neighbouring information needed to determine the stencil supports $\Xi_{\zeta}$, and it helps to extend the solution $\hat{u}$ from the discrete set of nodes $\Xi$ to any points in the domain. In a meshless method one has to provide meshless algorithms for all these goals. See [17] for a discussion of various aspects of giving up a mesh in meshless methods. We will not address here the extension of $\hat{u}$, which is a scattered data fitting problem. The problem of stencil support selection will be considered in Sections 4 and 5, and the problem of domain discretisation in an adaptive manner will be addressed in Section 6. Before that, we describe in the next section three methods of computing stencil coefficients using RBF interpolation.

\section{Stencil coefficients from RBF interpolation}

Assuming $\Xi_{\zeta}$ and $\Theta_{\zeta}$ have been chosen, radial basis function interpolation methods can be used to generate the stencil coefficients $\sigma_{\zeta, \theta}$ and $w_{\zeta, \xi}$ as the weights of a numerical differentiation formula

$$
\sum_{\theta \in \Theta_{\zeta}} \sigma_{\zeta, \theta} \Delta u(\theta) \approx \sum_{\xi \in \Xi_{\zeta}} w_{\zeta, \xi} u(\xi)
$$

In this section we discuss three methods for determining stencil coefficients, called single point RBF stencil, multipoint RBF stencil and Hermite RBF stencil. In each case we assume that $\Xi$ and $\Xi_{\zeta}, \zeta \in \Xi \backslash \partial \Xi$, are known and describe how $\Theta_{\zeta}$ and the weights $\sigma_{\zeta, \theta}$ and $w_{\zeta, \xi}$ are computed. Then $\Theta$ is defined by $\Theta:=\bigcup_{\zeta \in \Xi \backslash \partial \Xi} \Theta_{\zeta}$. 
Let the function $\phi: \mathbb{R}_{+} \rightarrow \mathbb{R}$ be positive definite or conditionally positive definite function of order 1 . Given any set $\Xi=\left\{\xi_{1}, \ldots, \xi_{n}\right\} \subset \mathbb{R}^{d}$ and a function $u: \mathbb{R}^{d} \rightarrow \mathbb{R}$, the $R B F$ interpolant $s$ is sought in the form

$$
\begin{aligned}
& s(x)=\sum_{j=1}^{n} a_{j} \Phi\left(x-\xi_{j}\right)+c, \quad \Phi(x):=\phi(\|x\|) \\
& s\left(\xi_{i}\right)=u\left(\xi_{i}\right), \quad i=1, \ldots, n,
\end{aligned}
$$

where $c$ is a constant. Then the coefficients $a_{j}$ and $c$ are uniquely determined by the following conditions [43]

$$
\begin{aligned}
\sum_{j=1}^{n} a_{j} \Phi\left(\xi_{i}-\xi_{j}\right)+c & =u\left(\xi_{i}\right), \quad i=1, \ldots, n \\
\sum_{j=1}^{n} a_{j} & =0
\end{aligned}
$$

which can be written in matrix form as the linear equation

$$
\left[\begin{array}{ll}
\Phi_{\Xi} & \mathbf{1} \\
\mathbf{1}^{T} & 0
\end{array}\right]\left[\begin{array}{l}
a \\
c
\end{array}\right]=\left[\begin{array}{c}
u_{\mid \Xi} \\
0
\end{array}\right], \quad \Phi_{\Xi}:=\left[\Phi\left(\xi_{i}-\xi_{j}\right)\right]_{i, j=1}^{n}, \quad \mathbf{1}:=\left[\begin{array}{lll}
1 & \cdots & 1
\end{array}\right]^{T},
$$

with a symmetric positive definite matrix.

Note that both $c$ and the side condition $\sum_{j=1}^{n} a_{j}=0$ can be removed in the above if $\phi$ is positive definite (and for certain conditionally positive definite functions like multiquadric $\phi(r)=\sqrt{1+r^{2}}$ ), or $c$ can be replaced by a higher degree polynomial, leading to a more complicated side condition and certain restrictions on the location of points $\xi_{j}$. In the case of a higher degree polynomial, conditionally positive definite functions of higher orders can be used, for example the thin plate spline $\phi(r)=r^{2} \log r$ which is a conditionally positive definite function of order 2 and hence requires at least a linear polynomial term.

In this paper we only consider the RBF interpolant with a constant term, which is indeed a popular choice in particular because it does not introduce any restrictions on $\Xi$ and the interpolant reproduces constants exactly. Several functions $\phi$ that are suitable for this setting are known explicitely [43], but we restrict our attention to following three positive definite basis functions that have shown a good performance in our numerical tests:

$$
\operatorname{Gaussian}(\mathrm{G}) \quad \phi_{\mathrm{g}}(r)=e^{-r^{2}}
$$

inverse multiquadric (IMQ) $\quad \phi_{\text {imq }}(r)=1 / \sqrt{1+r^{2}}$

$$
\text { Wendland's } C^{6} \text { function (W33) } \quad \phi_{\mathrm{w} 33}(r)=(1-r)_{+}^{8}\left(32 r^{3}+25 r^{2}+8 r+1\right)
$$

As a function $\phi(r)$ remains positive definite when $r$ is scaled, a scaling parameter $\delta>0$ is introduced, and the radial basis function $\phi$ in (5) takes the form $\phi(r)=\phi_{\mathrm{g}}(r / \delta)$, $\phi(r)=\phi_{\text {imq }}(r / \delta)$ or $\phi(r)=\phi_{\mathrm{w} 33}(r / \delta)$.

RBF interpolant $s(x)$ provides a good approximation of $u(x)$ if the function $u$ is sufficiently smooth and the set of points $\xi_{1}, \ldots, \xi_{n} \in \mathbb{R}^{d}$ is sufficiently dense in a neighbourhood of $x$ [43]. Moreover, the derivatives of $s$ are good approximations of the 
derivatives of $u$ if $\phi$ is sufficiently smooth. Therefore an approximation of $D u(x)$, where $D$ is a linear differential operator annihilating constants, may be considered in the form

$$
D u(x) \approx D s(x)=\sum_{j=1}^{n} a_{j} D \Phi\left(x-\xi_{j}\right)=\sum_{i=1}^{n} w_{i} u\left(\xi_{i}\right),
$$

where the weights $w_{i}$ (depending on $x$ ) exist because the coefficients $a_{j}$ of the interpolation function $s$ defined by (5)-(6) depend linearly on the data $u\left(\xi_{i}\right), i=1, \ldots, n$. These weights can be found by solving the symmetric positive definite linear system

$$
\left[\begin{array}{ll}
\Phi_{\Xi} & \mathbf{1} \\
\mathbf{1}^{T} & 0
\end{array}\right]\left[\begin{array}{l}
w \\
v
\end{array}\right]=\left[\begin{array}{c}
D \Phi(x-\cdot)_{\mid \Xi} \\
0
\end{array}\right],
$$

that is by solving the RBF interpolation problem (5)-(6) with the data given by $D \Phi(x-$ $\left.\xi_{i}\right), i=1, \ldots, n$. Indeed, if the weight vector $w$ satisfies (8), then

$$
\begin{aligned}
\sum_{j=1}^{n} a_{j} D \Phi\left(x-\xi_{j}\right) & =\left[\begin{array}{c}
a \\
c
\end{array}\right]^{T}\left[\begin{array}{c}
D \Phi(x-\cdot)_{\mid \Xi} \\
0
\end{array}\right]=\left[\begin{array}{l}
a \\
c
\end{array}\right]^{T}\left[\begin{array}{ll}
\Phi_{\Xi} & \mathbf{1} \\
\mathbf{1}^{T} & 0
\end{array}\right]\left[\begin{array}{l}
w \\
v
\end{array}\right] \\
& =\left[\begin{array}{c}
u_{\mid \Xi} \\
0
\end{array}\right]^{T}\left[\begin{array}{l}
w \\
v
\end{array}\right]=\sum_{i=1}^{n} w_{i} u\left(\xi_{i}\right) .
\end{aligned}
$$

We now make use of the weights (8) to define two types of RBF stencils for the discretisation of the Dirichlet problem (1) in the form (2).

Single point RBF stencil. Similar to the finite difference stencils, a numerical differentiation formula for the value of the Laplacian of $u$ at a single point $\zeta$ is used, and hence $\Theta_{\zeta}=\{\zeta\}, \sigma_{\zeta, \zeta}=1$ in (2). Given a local set $\Xi_{\zeta}$ of discretisation centres, the weight vector $w=\left[w_{\zeta, \xi}\right]_{\xi \in \Xi_{\zeta}}$ in (2) is computed from the linear system

$$
\left[\begin{array}{cc}
\Phi_{\Xi_{\zeta}} & \mathbf{1} \\
\mathbf{1}^{T} & 0
\end{array}\right]\left[\begin{array}{c}
w \\
v
\end{array}\right]=\left[\begin{array}{c}
\Delta \Phi(\zeta-\cdot)_{\mid \Xi_{\zeta}} \\
0
\end{array}\right] .
$$

Multipoint RBF stencil. Motivated by the stencils resulting from the finite element discretisation (see Section 2), we also consider a method of discretisation of the Dirichlet problem based on numerical differentiation of a linear combination of Laplacians

$$
D u=\sum_{\theta \in \Theta_{\zeta}} \sigma_{\zeta, \theta} \Delta u(\cdot-\zeta+\theta)
$$

Then $D u(\zeta)=\sum_{\theta \in \Theta_{\zeta}} \sigma_{\zeta, \theta} \Delta u(\theta)$, and the numerical differentiation formula (7),

$$
D u(\zeta) \approx \sum_{\xi \in \Xi_{\zeta}} w_{\zeta, \xi} u(\xi)
$$

has the form (4) and leads to (2) with the weight vector $w=\left[w_{\zeta, \xi}\right]_{\xi \in \Xi_{\zeta}}$ computed from

$$
\left[\begin{array}{cc}
\Phi_{\Xi_{\zeta}} & \mathbf{1} \\
\mathbf{1}^{T} & 0
\end{array}\right]\left[\begin{array}{c}
w \\
v
\end{array}\right]=\left[\begin{array}{c}
\sum_{\theta \in \Theta_{\zeta}} \sigma_{\zeta, \theta} \Delta \Phi(\theta-\cdot)_{\mid \Xi_{\zeta}} \\
0
\end{array}\right] .
$$


Although various choices for $\Theta_{\zeta}$ and $\sigma_{\zeta, \theta}$ are possible, in this paper we take $\sigma_{\zeta, \theta}=1$ for all $\theta \in \Theta_{\zeta}$, and apply the following algorithm to determine $\Theta_{\zeta}$ : Given a set $\Xi_{\zeta}=\{\zeta=$ $\left.\xi_{1}, \xi_{2}, \ldots, \xi_{n}\right\} \subset \mathbb{R}^{2}$ such that $\zeta$ lies in the convex full $\Omega_{\zeta}$ of $\left\{\xi_{2}, \ldots, \xi_{n}\right\}$ and all rays $\zeta \xi_{i}$, $i=2, \ldots, n$ are different, split $\Omega_{\zeta}$ into $n-1$ triangles $T_{1}, \ldots, T_{n-1}$ with vertices in $\Xi_{\zeta}$ sharing a common vertex $\zeta$, and define $\Theta_{\zeta}=\left\{\theta_{1}, \ldots, \theta_{n-1}\right\}$ to be the set of barycentres of $T_{1}, \ldots, T_{n-1}$. For a set $\Xi_{\zeta} \subset \mathbb{R}^{3}$ a similar splitting into tetrahedra can be used, where the tetrahedra are determined by the triangles of a Delaunay triangulation on the unit sphere centred at $\zeta$ of the intersection points of the rays $\zeta \xi_{i}, i=2, \ldots, n$ with this sphere.

Note that the above $\Theta_{\zeta}$ might look unusual from the point of view of numerical differentiation because $\Theta_{\zeta}$ does not contain $\zeta$. Moreover, it mimics the finite element 'stencil' which apparently does not stem from any numerical differentiation method, and so a priori it is not obvious that a similar stencil generated by numerical differentiation should be good.

As shown in [44], numerical differentiation formulas of the type (4) can also be generated with the help of the RBF Hermite interpolant $s_{\mathrm{H}}$ [43, Chapter 16], which we only formulate in the case of a single Laplacian and a constant polynomial term, even though more general settings are also possible,

$$
\begin{aligned}
s_{\mathrm{H}}(x) & =\sum_{j=1}^{n} a_{j} \Phi\left(x-\xi_{j}\right)+\sum_{j=1}^{m} b_{j} \Delta \Phi\left(x-\theta_{j}\right)+c, \quad \sum_{j=1}^{n} a_{j}=0, \\
s_{\mathrm{H}}\left(\xi_{i}\right) & =u\left(\xi_{i}\right), \quad i=1, \ldots, n, \\
\Delta s_{\mathrm{H}}\left(\xi_{i}\right) & =\Delta u\left(\theta_{i}\right), \quad i=1, \ldots, m,
\end{aligned}
$$

where, as before, $c$ is a constant and $\Phi(x)=\phi(\|x\|)$ with $\phi$ sufficiently smooth and positive definite or conditionally positive definite of order 1 . If $\Phi \in L^{1}\left(\mathbb{R}^{d}\right) \cup C^{4}\left(\mathbb{R}^{d}\right)$, then the solution of the above Hermite interpolation problem exists and is unique for any data $u\left(\xi_{i}\right), i=1, \ldots, n, \Delta u\left(\theta_{i}\right), i=1, \ldots, m$, as soon as each $\Xi=\left\{\xi_{1} \ldots, \xi_{n}\right\}$ and $\Theta=\left\{\theta_{1} \ldots, \theta_{m}\right\}$ consists of distinct points, even though $\Xi$ and $\Theta$ may coincide or overlap. This follows from [43, Theorem 16.5] if one takes into account that the assumptions of this theorem may be verified similar to the proof of [43, Theorem 16.4]. Note that $\Phi \in L^{1}\left(\mathbb{R}^{d}\right) \cup C^{4}\left(\mathbb{R}^{d}\right)$ for two of the radial basis functions $\phi$ used in this paper: $\phi_{\mathrm{g}}$ and $\phi_{\mathrm{w} 33}$. Inverse multiquadric $\phi_{\mathrm{imq}}$ is infinitely differentiable but leads to $\Phi \notin L^{1}\left(\mathbb{R}^{d}\right)$, which makes it impossible to use the above mentioned results of [43] but does not seem to cause any problem in numerical examples below.

The coefficients $\left\{a_{j}\right\},\left\{b_{j}\right\}, c$ of $s_{\mathrm{H}}$ are determined from the symmetric positive definite linear system

$$
\left[\begin{array}{ccc}
\Phi_{\Xi} & \Delta \Phi_{\Xi, \Theta} & \mathbf{1} \\
\Delta \Phi_{\Xi}^{T}, \Theta & \Delta^{2} \Phi_{\Theta} & 0 \\
\mathbf{1}^{T} & 0 & 0
\end{array}\right]\left[\begin{array}{l}
a \\
b \\
c
\end{array}\right]=\left[\begin{array}{c}
u_{\mid \Xi} \\
\Delta u_{\mid \Theta} \\
0
\end{array}\right]
$$

where $\Phi_{\Xi}$ is defined as before, and

$$
\Delta \Phi_{\Xi, \Theta}:=\left[\Delta \Phi\left(\xi_{i}-\theta_{j}\right)\right]_{i=1, j=1}^{n, m}, \quad \Delta^{2} \Phi_{\Theta}:=\left[\Delta^{2} \Phi\left(\theta_{i}-\theta_{j}\right)\right]_{i, j=1}^{m} .
$$


RBF Hermite interpolant can be used to estimate the Laplacian of $u$ at a point $x$ as

$$
\begin{aligned}
\Delta u(x) \approx \Delta s_{\mathrm{H}}(x) & =\sum_{j=1}^{n} a_{j} \Delta \Phi\left(x-\xi_{j}\right)+\sum_{j=1}^{m} b_{j} \Delta^{2} \Phi\left(x-\theta_{j}\right) \\
& =\sum_{i=1}^{n} w_{i} u\left(\xi_{i}\right)+\sum_{i=1}^{m} \sigma_{i} \Delta u\left(\theta_{i}\right),
\end{aligned}
$$

where the weights $\left\{w_{i}\right\}$ and $\left\{\sigma_{i}\right\}$ are found by solving the linear system

$$
\left[\begin{array}{ccc}
\Phi_{\Xi} & \Delta \Phi_{\Xi, \Theta} & \mathbf{1} \\
\Delta \Phi_{\Xi}^{T}, \Theta & \Delta^{2} \Phi_{\Theta} & 0 \\
\mathbf{1}^{T} & 0 & 0
\end{array}\right]\left[\begin{array}{c}
w \\
\sigma \\
v
\end{array}\right]=\left[\begin{array}{c}
\Delta \Phi(x-\cdot)_{\mid \Xi} \\
\Delta^{2} \Phi(x-\cdot)_{\mid \Theta} \\
0
\end{array}\right]
$$

see [44]. The above numerical differentiation formula is now written in the form (4),

$$
\Delta u\left(\theta_{0}\right)-\sum_{i=1}^{m} \sigma_{i} \Delta u\left(\theta_{i}\right) \approx \sum_{i=1}^{n} w_{i} u\left(\xi_{i}\right), \quad \theta_{0}:=x
$$

which leads to a discretisation equation in the form (2). This approach resembles Collatz' Mehrstellenverfahren in the finite difference method. It is proposed in [44] to take $\theta_{0}=\xi_{1}=\zeta$, the central point of the stencil, and choose $\Theta \subseteq \Xi$ such that certain diagonal dominance criterion is met. Since there is little hope that the diagonal dominance condition can always be fulfilled on adaptive centres, we choose $m=n-1$ and $\theta_{i}=\xi_{i+1}$, $i=0,1, \ldots, n-1$, which corresponds to the highest consistency order according to the numerical tests in [44, Section 4.3]. Finally, since the row $\left[\Delta \Phi_{\Xi, \Theta}^{T} \Delta^{2} \Phi_{\Theta} 0\right]$ of the matrix in (11) involves second order derivatives of $\Phi$, we scale it and the corresponding column by $d^{2}$, where $d=d\left(\Xi_{\zeta}\right):=\max \left\{\|\zeta-\xi\|: \xi \in \Xi_{\zeta}\right\}$ to precondition the matrix. This results in the following method.

Hermite RBF stencil. Given a local set $\Xi_{\zeta}$ of discretisation centres containing $\zeta$, choose $\Theta_{\zeta}=\Xi_{\zeta}$ and set $\tilde{\Theta}_{\zeta}:=\Theta_{\zeta} \backslash\{\zeta\}$. The weights in (2) are determined as follows. Set $\sigma_{\zeta, \zeta}=1$ and $\sigma_{\zeta, \theta}=-\tilde{\sigma}_{\zeta, \theta}$ for all $\theta \in \tilde{\Theta}_{\zeta}$, where the vector $\tilde{\sigma}=\left[\tilde{\sigma}_{\zeta, \theta}\right]_{\theta \in \tilde{\Theta}_{\zeta}}$, together with the other weight vector $w=\left[w_{\zeta, \xi}\right]_{\xi \in \Xi_{\zeta}}$ are computed from the linear system

$$
\left[\begin{array}{ccc}
\Phi_{\Xi_{\zeta}} & d^{2} \Delta \Phi_{\Xi_{\zeta}, \tilde{\Theta}_{\zeta}} & \mathbf{1} \\
d^{2} \Delta \Phi_{\Xi_{\zeta}, \tilde{\Theta}_{\zeta}}^{T} & d^{4} \Delta^{2} \Phi_{\tilde{\Theta}_{\zeta}} & 0 \\
\mathbf{1}^{T} & 0 & 0
\end{array}\right]\left[\begin{array}{c}
w \\
d^{-2} \tilde{\sigma} \\
v
\end{array}\right]=\left[\begin{array}{c}
\Delta \Phi(\zeta-\cdot)_{\mid \Xi_{\zeta}} \\
d^{2} \Delta^{2} \Phi(\zeta-\cdot)_{\mid \tilde{\Theta}_{\zeta}} \\
0
\end{array}\right] .
$$

For each of the above RBF stencil methods, a discretisation of the Dirichlet problem as described in Section 2 will be complete as soon as two algorithms are provided: (a) an algorithm to generate the set $\Xi$ of discretisation centres, and (b) an algorithm to select the local sets $\Xi_{\zeta} \subset \Xi$ for each interior centre $\zeta \in \Xi \backslash \partial \Xi$. These algorithms will be addressed in Sections 4-6. 
Choice of scaling parameter. It is well known that the quality of the RBF interpolation heavily depends on the scaling parameter $\delta>0$ in $\phi(r)=\phi_{\mathrm{g}}(r / \delta), \phi_{\text {imq }}(r / \delta)$ or $\phi_{\mathrm{w} 33}(r / \delta)$. Therefore, methods for determining its optimal values have been intensively investigated, see [15, Chapters 16 and 17]. In particular, there is a trade-off between the accuracy of the interpolant which improves when $\delta$ increases, and the conditioning of the interpolation matrix $\Phi_{\Xi}$ which deteriorates in the same time [37]. Methods based on asymptotic expansion of the analytic RBFs with respect to the powers of $1 / \delta$ presented in [44] allow stable computation of the RBF stencils even with extremely large $\delta$, leading to potentially very accurate numerical differentiation. In this paper we do not explore these promising techniques, nor do we try to use the quadruple precision computations to overcome the ill-conditioning of the RBF matrices as in [42]. Our preliminary experiments have confirmed the observations from the earlier literature that the best results are obtained if $\delta$ is chosen as large as possible with the RBF matrix still numerically non-singular. Therefore, for each $\Xi_{\zeta}$ we compute $\delta$ as large as possible with the condition number of the matrix of (9), (10) or (12) not exceeding $10^{12}$. This value of $\delta$ is found by a bisection type method with the starting interval $[0.1,400]$ for $\delta / d\left(\Xi_{\zeta}\right)$, and the condition number is estimated using the singular value decomposition.

We now discuss a numerical experiment that compares the errors of the solutions of the Dirichlet problem obtained using the three types of RBF stencils on a uniform grid.

Test Problem 1. Consider Dirichlet problem (1) with homogeneous boundary conditions $g=0$, where the domain $\Omega$ is the square $(0,1)^{2}$, the right hand side is defined by $f(x, y)=-2 \pi^{2} \sin \pi x \sin \pi y$. The exact solution is $u(x, y)=\sin \pi x \sin \pi y$.

For a number of values of $N$ between $N=6$ and $N=44$, we defined the set discretisation centres $\Xi$ as the nodes of the uniform $N \times N$ grid in [0,1]. The stencil supports $\Xi_{\zeta}, \zeta \in \Xi \backslash \partial \Xi$, are the 5 grid points as in the standard 5 point stencil used in the finite difference method. We used scaled Gaussian RBFs $\phi_{\mathrm{g}}(r / \delta)$ to determine the single point, multipoint and Hermite RBF stencil coefficients. The above method of choosing scaling parameter led to the values $\delta / d\left(\Xi_{\zeta}\right) \approx 720$ for both single point and multipoint stencils, and $\delta / d\left(\Xi_{\zeta}\right) \approx 31$ for the Hermite stencil (independent of $N$ ).

Fig. 1(a) shows the normalised root mean square (rms) error of the numerical differentiation formula (4), computed as

$$
(\# \Xi \backslash \partial \Xi)^{-1}\left(\sum_{\zeta \in \Xi \backslash \partial \Xi} r_{\zeta}^{2}\right)^{1 / 2}, \quad r_{\zeta}:=\left(\sum_{\theta \in \Theta_{\zeta}} \sigma_{\zeta, \theta} \Delta u(\theta)-\sum_{\xi \in \Xi_{\zeta}} w_{\zeta, \xi} u(\xi)\right) / \sum_{\theta \in \Theta_{\zeta}}\left|\sigma_{\zeta, \theta}\right|,
$$

whereas Fig. 1(b) presents the rms error of the solution $\hat{u}$ of (2) against the exact solution $u$,

$$
(\# \Xi \backslash \partial \Xi)^{-1}\left(\sum_{\zeta \in \Xi \backslash \partial \Xi}\left(\hat{u}_{\zeta}-u(\zeta)\right)^{2}\right)^{1 / 2} .
$$

As we can see in these figures, there is a strong correlation between numerical differentiation error and the error of the solution of the PDE. The errors in case of the single point RBF stencil is very close to the error of the finite difference solution. This is not surprising as the single point Gaussian stencil is known to converge to the finite difference 5 point stencil as $\delta \rightarrow \infty[44]$. Both multipoint and Hermite stencils exhibit up to 


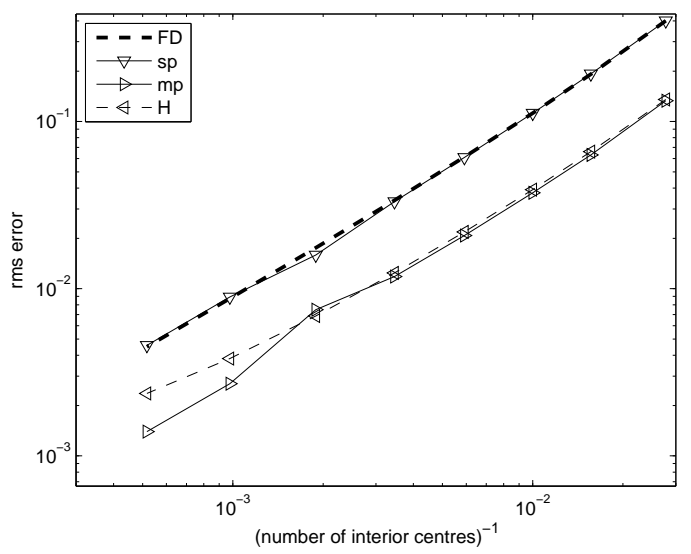

(a) Numerical differentiation error

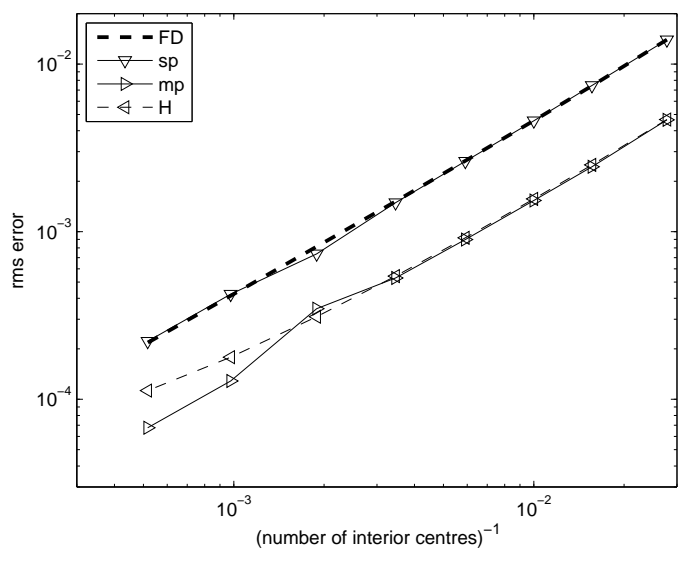

(b) Dirichlet problem error

Figure 1: RBF solution with Gaussian $\phi_{\mathrm{g}}$ on a grid using 5 point stencil supports for Test Problem 1. Root mean square (rms) numerical differentiation error (a) and rms error of the solution of the Dirichlet problem (b) are shown for the three types of RBF stencils in comparison with the finite difference method. Legend: FD refers the finite difference method with the standard 5 point stencil; sp, mp and $\mathrm{H}$ refer to the single point, multipoint and Hermite RBF stencils, respectively.

5 times better errors in this test. Comparing the slopes of the curves, we conjecture the $h^{2}$ convergence order for all three RBF methods if a fixed scaling parameter $\delta$, rather than the one proportional to $d\left(\Xi_{\zeta}\right)$, can be used. These numerical results complement those in $[42,44]$.

\section{RBF stencils on adaptive FEM meshes}

In this section we study the performance of RBF stencils in the following setting: The discretisation centres $\Xi$ and stencil supports $\Xi_{\zeta}$ are generated by the linear triangle finite element method with midpoint quadrature, as described in Section 2. The sets $\Theta, \Theta_{\zeta}$ and coefficients $\sigma_{\zeta, \theta}, w_{\zeta, \xi}$ are then chosen according to the single point (sp), multipoint (mp) and Hermite (h) RBF methods of Section 3.

In the case that $\Xi$ is a uniform or quasi-uniform mesh, several numerical examples in [42] show that the accuracy of the solution of (2) based on the single point RBF stencils is comparable with (and often significantly better than) the results obtained with the FEM stencils. Our own experiments only confirm this observation, and we do not present them here.

Our main interest are adaptively generated centres, and in this section they are the nodes of an adaptive mesh. We consider a test problem, where adaptive finite element meshes are known to perform much better than quasi-uniform meshes.

Test Problem 2. [1, function adaptmesh] Domain $\Omega$ is the circle sector given by the inequalities $r<1,-3 \pi / 4<\varphi<3 \pi / 4$ in polar coordinates, the right hand side $f=0$, the boundary conditions are defined by $g(r, \varphi)=\cos (2 \varphi / 3)$ along the arc, and $g(r, \varphi)=0$ along the straight lines. The exact solution of $(1)$ is $u(r, \varphi)=r^{2 / 3} \cos (2 \varphi / 3)$. 
MATLAB PDE Toolbox code provided in [1] (available also via MATLAB help entry on adaptmesh) generates a sequence of adaptive triangulations of $\Omega$, where more refinements are made near the singularity of the exact solution, resulting in a denser distribution of the centres in the vicinity of the origin, as shown in Fig. 2. For each triangulation generated by this code we place the centres $\Xi$ at the vertices of the triangulation and for each $\zeta \in \Xi \backslash \partial \Xi$ select the sets $\Xi_{\zeta}$ as in the finite element methods, e.g. by combining the vertices of all triangles attached to $\zeta$. Then we choose $\Theta, \Theta_{\zeta}, \sigma_{\zeta, \theta}$, $w_{\zeta, \xi}$ according to the three RBF methods presented in Section 3.
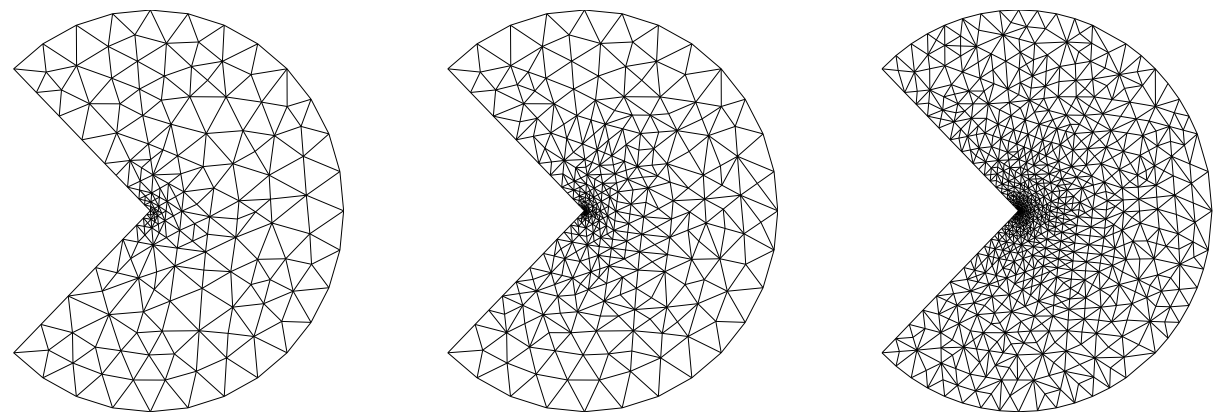

Figure 2: Adaptive triangulations for Test Problem 2 produced by the adaptive finite element method.

The rms errors (13) of the RBF solutions are presented in Fig. 3(a)-3(c) and compared to the rms error of the FEM solution. The results show that the slope of the error curves for all single point and multipoint stencils, as well as for the Hermite stencil in the case of $\phi_{\mathrm{w} 33}$ is roughly the same as the slope of the error curve obtained with the finite element method, which indicates the same convergence order. However, the distance between the curves shows that the errors of the RBF methods are 2-4 times higher. Much less stable error curves are obtained with Hermite RBF stencil when the functions $\phi_{\mathrm{g}}$ are $\phi_{\mathrm{imq}}$ are used (see Fig. 3(c)). This suggests that compactly supported RBFs should be preferable when using Hermite RBF stencils. Finally, Fig. 3(d) presents the minimum relative scaling in the RBF tests, computed as

$$
\min _{\zeta \in \Xi \backslash \partial \Xi} \delta_{\zeta} / d\left(\Xi_{\zeta}\right)
$$

where $\delta_{\zeta}$ is the value of the scaling parameter used in the stencil computation at $\zeta$ (see the comments at the end of Section 3), and $d\left(\Xi_{\zeta}\right)=\max \left\{\|\zeta-\xi\|: \xi \in \Xi_{\zeta}\right\}$. All curves are nearly constant, which indicates that it might be possible to empirically determine suitable values of the relative scaling parameter for each RBF and stencil type without the costly calculation of $\delta$ for each $\Xi_{\zeta}$. Note that the behaviour of the scaling parameter in our further tests in Sections 5 and 6 is quite similar to the one in Fig. 3(d).

\section{$5 \quad$ Meshless stencil support selection}

The approach of the previous section is clearly mesh dependent as it relies on the triangulation generated by the finite element method, even though RBF stencil weights 


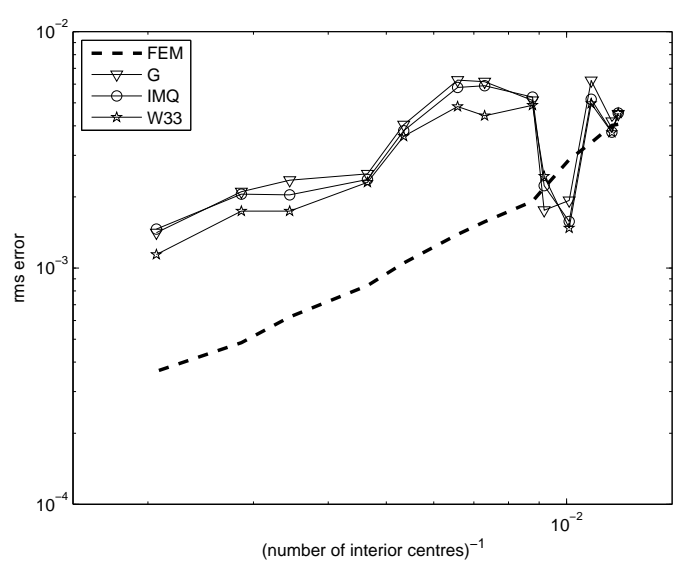

(a) Single point stencils

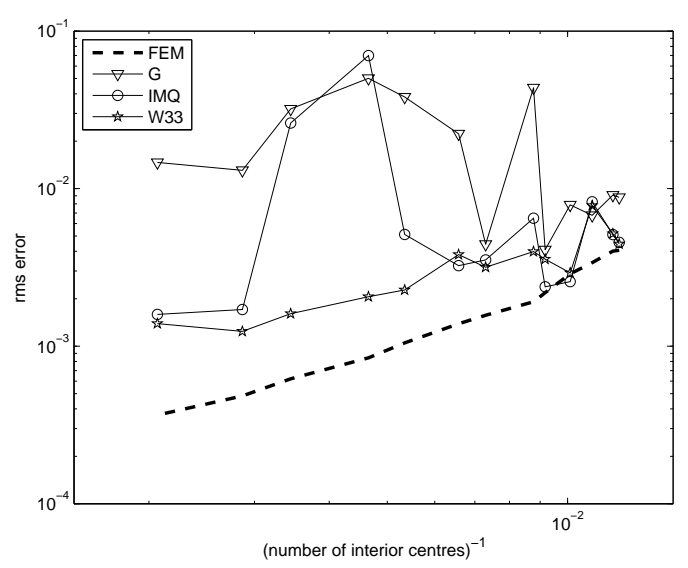

(c) Hermite stencils

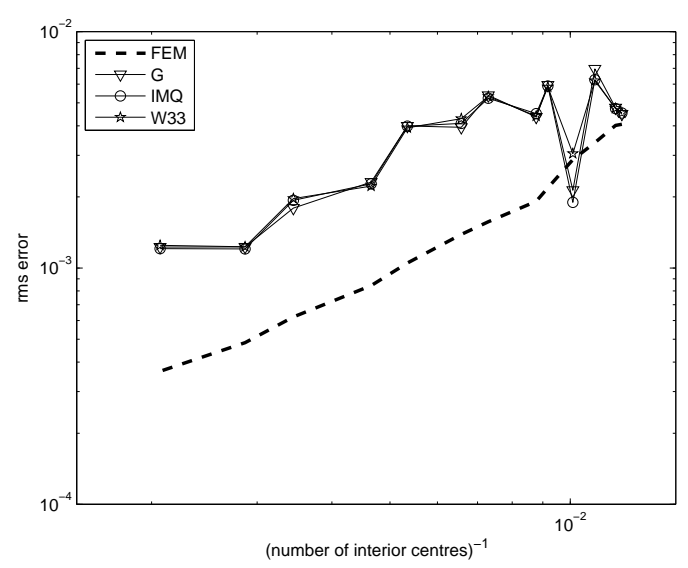

(b) Multipoint stencils

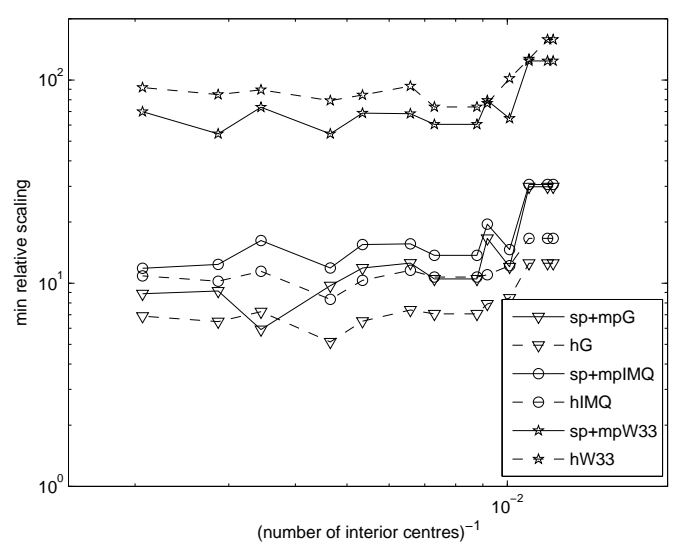

(d) Minimum relative scaling

Figure 3: RBF solution on adaptive FEM centres using FEM stencil supports $\Xi_{\zeta}$ for Test Problem 2. Figures (a)-(c) show the error obtained with three types of RBF stencils, whereas $(d)$ presents the minimum value of the scaling parameter relative to the stencil size. Legend: FEM refers the finite element method; G, IMQ and W33 refer to RBF stencils based on $\phi_{\mathrm{g}}, \phi_{\mathrm{imq}}$ and $\phi_{\mathrm{w} 33}$, respectively; the meaning of $\mathrm{sp}, \mathrm{mp}$ and $\mathrm{H}$ is the same as in Fig. 1. In (d) the graphs for the single point and multipoint stencils are combined because they turned out to be indistinguishable. 
are used. In this section we propose a meshless method for the selection of the stencil supports $\Xi_{\zeta}$ for the bivariate Dirichlet problem, and use the same Test Problem 2 to investigate its numerical performance and compare it with the performance of certain algorithms known in the literature. It is remarkable that stencil supports selected by this method turn out to be better on FEM centres than the 'native' FEM stencil supports, compare Figs 3 and 4.

Given $\zeta \in \Xi \backslash \partial \Xi$, we will select a set $\Xi_{\zeta} \subset \Xi$ containing $\zeta$. Let $\Xi_{\zeta}=\left\{\zeta, \xi_{1}, \ldots, \xi_{k}\right\}$, where the points $\xi_{1}, \ldots, \xi_{k}$ are ordered counterclockwise with respect to $\zeta$. Consider the following cost function

$$
\mu\left(\xi_{1}, \ldots, \xi_{k}\right):=\sum_{i=1}^{k} \alpha_{i}^{2},
$$

where $\alpha_{i}$ denotes the angle between the rays $\zeta \xi_{i}, \zeta \xi_{i+1}$ in the counterclockwise direction, with the cyclic identification $\xi_{k+i}:=\xi_{i}$. We will also need the minimum and the maximum angle

$$
\underline{\alpha}\left(\xi_{1}, \ldots, \xi_{k}\right)=\min \left\{\alpha_{1}, \ldots, \alpha_{k}\right\}, \quad \bar{\alpha}\left(\xi_{1}, \ldots, \xi_{k}\right)=\max \left\{\alpha_{1}, \ldots, \alpha_{k}\right\} .
$$

Since $\sum_{i=1}^{k} \alpha_{i}=2 \pi$, the expression $\sum_{i=1}^{k} \alpha_{i}^{2}$ would achieve its unique minimum for $\alpha_{1}=\cdots=\alpha_{k}=2 \pi / k$, that is for the uniformly spaces directions $\zeta \xi_{i}$ if $\xi_{1}, \ldots, \xi_{k}$ were chosen freely in $\mathbb{R}^{2}$. However, these points have to be in the prescribed set $\Xi$, and so the goal of the algorithm below is to choose $\xi_{1}, \ldots, \xi_{k} \in \Xi$ such that $\mu\left(\xi_{1}, \ldots, \xi_{k}\right)$ is minimised while keeping the distances $\left\|\xi_{i}-\zeta\right\|$ as small as possible. To achieve a balance between the goals of a small $\mu$ and small distances, we introduce the restriction that $\xi_{i}$ must be among $m$ closest points to $\zeta$, and terminate the algorithm if the set $\left\{\zeta, \xi_{1}, \ldots, \xi_{k}\right\}$ satisfies

$$
\bar{\alpha}\left(\xi_{1}, \ldots, \xi_{k}\right) \leq u \underline{\alpha}\left(\xi_{1}, \ldots, \xi_{k}\right),
$$

where $m>k$ and $u>1.0$ are parameters to be determined empirically.

\section{Algorithm 1. Meshless stencil support selection}

Input: $\Xi, \zeta$. Output: $\Xi_{\zeta}$. Parameters: $k$ (the target number of points $\xi_{i}$ ), $m>k$ (the number of points in the local cloud) and $u>1$ (the angle uniformity tolerance). Parameter values used in our numerical experiments: $k=6, m=30, u=3.0$.

I. Find $m$ nearest points $\xi_{1}, \ldots, \xi_{m}$ in $\Xi \backslash\{\zeta\}$ to $\zeta$, sorted by increasing distance to $\zeta$, and initialise $\Xi_{\zeta}:=\left\{\zeta, \xi_{1}, \ldots, \xi_{k}\right\}$. If $\bar{\alpha}\left(\xi_{1}, \ldots, \xi_{k}\right) \leq u \underline{\alpha}\left(\xi_{1}, \ldots, \xi_{k}\right)$, then STOP: return $\Xi_{\zeta}$.

II. For $i=n+1, \ldots, m$ :

1. Compute the angles $\alpha_{1}^{\prime}, \ldots, \alpha_{k+1}^{\prime}$ formed by the extended set $\left\{\xi_{1}^{\prime}, \ldots, \xi_{k+1}^{\prime}\right\}=$ $\left\{\xi_{1}, \ldots, \xi_{k}, \xi_{i}\right\}$.

2. If both angles between $\zeta \xi_{i}$ and its two neighbouring rays are greater than the minimum angle $\underline{\alpha}^{\prime}:=\underline{\alpha}\left(\xi_{1}, \ldots, \xi_{k}, \xi_{i}\right)$ :

i. Find $j$ such that $\alpha_{j}^{\prime}=\underline{\alpha}^{\prime}$. Choose $p=j$ or $p=j+1$ depending on whether $\alpha_{j-1}^{\prime}<\alpha_{j+1}^{\prime}$ or $\alpha_{j-1}^{\prime} \geq \alpha_{j+1}^{\prime}$. 
ii. If $\mu\left(\left\{\xi_{1}^{\prime}, \ldots, \xi_{k+1}^{\prime}\right\} \backslash\left\{\xi_{p}^{\prime}\right\}\right)<\mu\left(\xi_{1}, \ldots, \xi_{k}\right)$ :
a. Update $\left\{\xi_{1}, \ldots, \xi_{k}\right\}=\left\{\xi_{1}^{\prime}, \ldots, \xi_{k+1}^{\prime}\right\} \backslash\left\{\xi_{p}^{\prime}\right\}$.
b. If $\bar{\alpha}\left(\xi_{1}, \ldots, \xi_{k}\right) \leq u \underline{\alpha}\left(\xi_{1}, \ldots, \xi_{k}\right)$
STOP: return the current set $\Xi_{\zeta}=\left\{\zeta, \xi_{1}, \ldots, \xi_{k}\right\}$.

III. Observe that $\bar{\alpha}\left(\xi_{1}, \ldots, \xi_{k}\right)>u \underline{\alpha}\left(\xi_{1}, \ldots, \xi_{k}\right)$ must hold for the current set $\Xi_{\zeta}=$ $\left\{\zeta, \xi_{1}, \ldots, \xi_{k}\right\}$ if the algorithm has not been terminated earlier. Find $j$ such that $\alpha_{j}=\underline{\alpha}\left(\xi_{1}, \ldots, \xi_{k}\right)$. Choose $p=j$ or $p=j+1$ depending on whether $\alpha_{j-1}<\alpha_{j+1}$ or $\alpha_{j-1} \geq \alpha_{j+1}$. STOP: return $\Xi_{\zeta}=\left\{\zeta, \xi_{1}, \ldots, \xi_{k}\right\} \backslash\left\{\xi_{p}\right\}$.

\section{Remarks}

1. If the algorithm terminates before Step III then $\Xi_{\zeta}$ consists of $k+1$ points (including $\zeta)$. Otherwise, the number of points is $k$. We choose $k=6$ to ensure that the matrix $\left[w_{\zeta, \xi}\right]_{\zeta, \xi \in \Xi \backslash \partial \Xi}$ of the linear system (2) resulting form RBF discretisation is about as sparse as the same matrix for the FEM discretisation, see Fig. 4(d) for the comparison of the density of the two matrices.

2. The $m$ closest points in Step I can be found efficiently in a meshless manner by using the standard space-partitioning data structures such as kd-tree.

3. it is easy to see that the choice of $p$ in Step II(2)i ensures that

$$
\mu\left(\left\{\xi_{1}^{\prime}, \ldots, \xi_{k+1}^{\prime}\right\} \backslash\left\{\xi_{p}^{\prime}\right\}\right)=\min \left\{\mu\left(\left\{\xi_{1}^{\prime}, \ldots, \xi_{k+1}^{\prime}\right\} \backslash\left\{\xi_{j}^{\prime}\right\}\right), \mu\left(\left\{\xi_{1}^{\prime}, \ldots, \xi_{k+1}^{\prime}\right\} \backslash\left\{\xi_{j+1}^{\prime}\right\}\right)\right\} .
$$

A similar observation applies to the choice of $p$ in Step III.

4. In case of complicated domains the points $\xi_{i}$ such that the segment between $\zeta$ and $\xi_{i}$ intersects domain boundary should be removed in Step I.

Fig. 4 presents the results of our numerical experiments for Test Problem 2, where the centres $\Xi$ are generated by adaptmesh as in Section 4 , but the stencil supports $\Xi_{\zeta}$ for the RBF methods are selected according to Algorithm 1. Figs 4(a)-(c) show that the discretisations obtained with the single point, multipoint and, in case of $\phi_{\mathrm{w} 33}$, Hermite RBF stencils often lead to the solutions of (1) with 2 to 3 times smaller rms error than the error of the FEM solution. In the same time, as seen in Fig. 4(d), the matrix

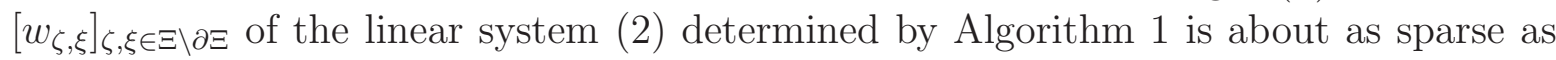
the matrix of the FEM discretisation.

For the sake of comparison, we implemented several stencil selection algorithms known from the literature and tested them on Test Problem 2 in the same setting as Algorithm 1 above. The results are presented in Fig. 5, where we only considered single point RBF stencil with $\phi_{\mathrm{g}}$ (or $\phi_{\mathrm{imq}}$ in two cases where its performance was better). As all error curves in Fig. 5(a) are above the reference FEM curve whereas the Algorithm 1 curves in Fig. 4(a) are below it, we conclude that Algorithm 1 compares favourably to all these methods. The density graph in Fig. 5(b) compared with Fig. 4(d) shows that only SLS leads to a significantly sparser linear system (2) than the one derived with Algorithm 1. 


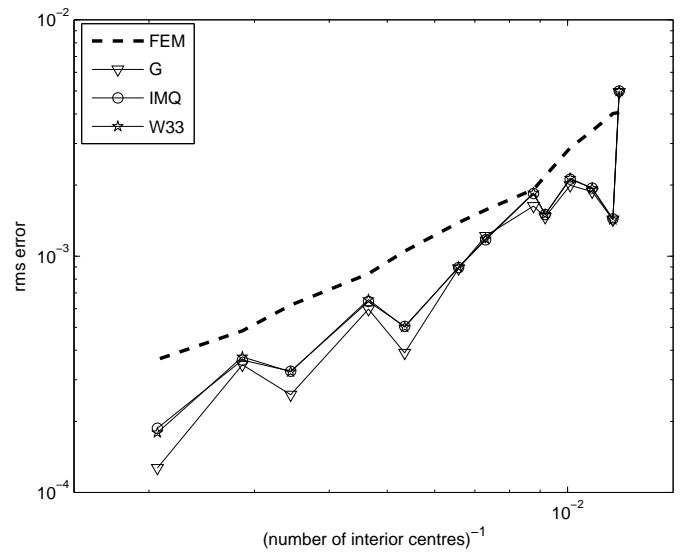

(a) Single point stencils

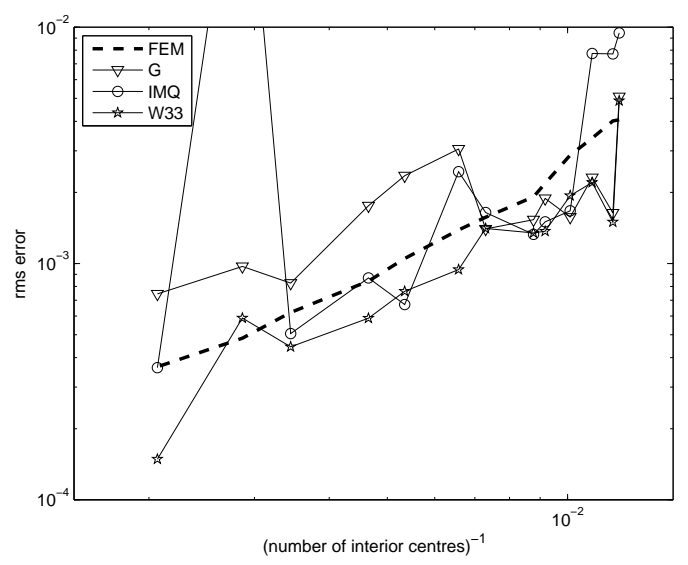

(c) Hermite stencils

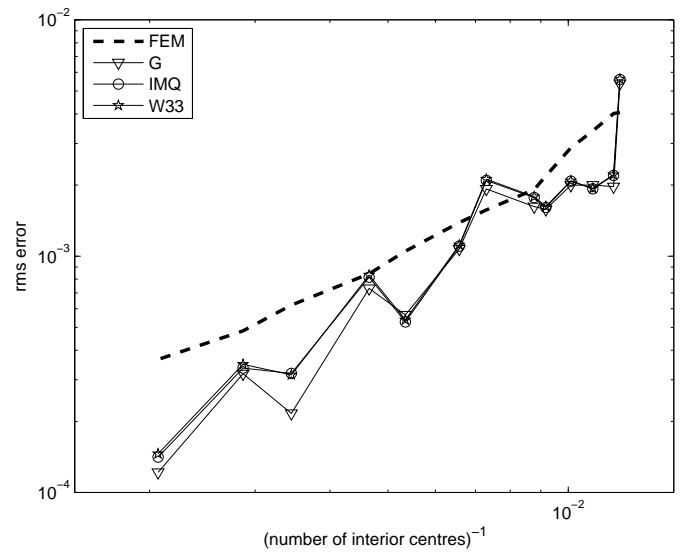

(b) Multipoint stencils

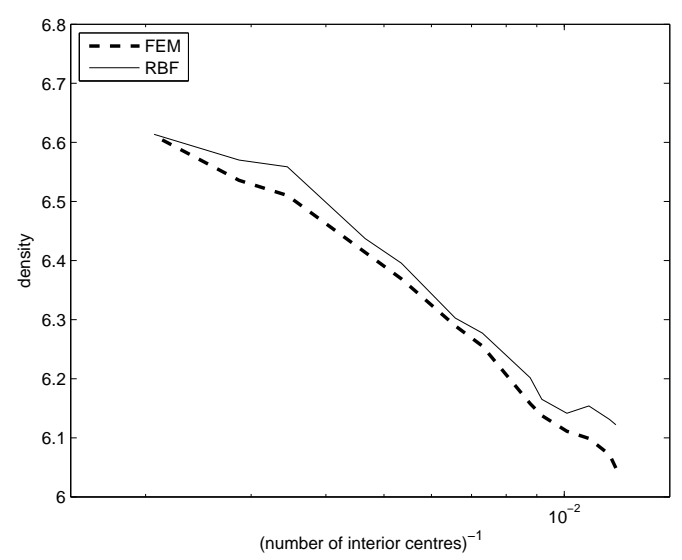

(d) Matrix density

Figure 4: RBF solution on adaptive FEM centres using RBF stencil supports according to Algorithm 1 for Test Problem 2. The first three graphs show the error obtained with three types of RBF stencils, whereas the forth graph presents the density of the sparse matrix of the linear system (2) for both FEM and RBF stencil support. The density (or bandwidth) of a matrix $A \in \mathbb{R}^{n \times n}$ is computed as $\operatorname{nnz}(A) / n$, where $\operatorname{nnz}(A)$ denotes the number of nonzero entries in $A$. 


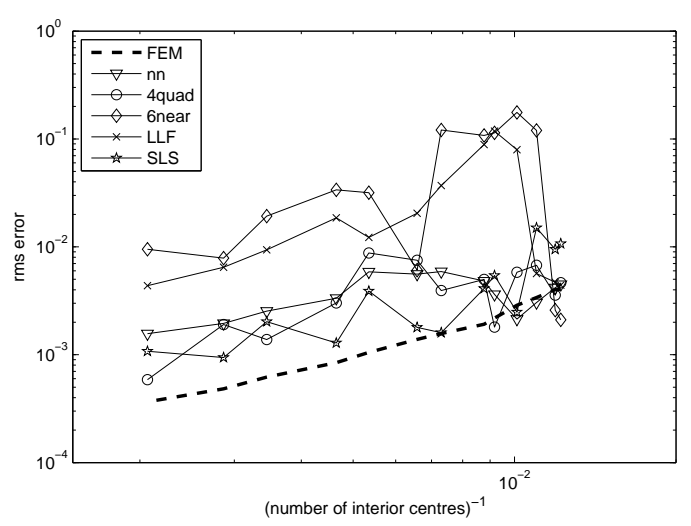

(a) RMS error

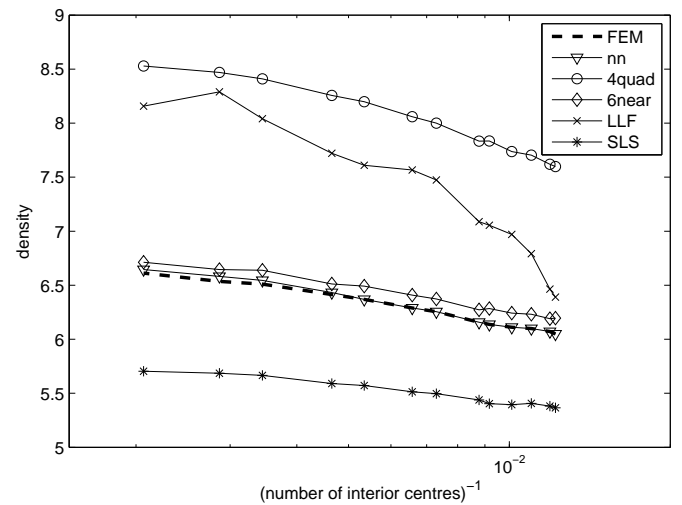

(b) Matrix density

Figure 5: RBF solution on adaptive FEM centres using RBF stencil supports obtained by the following algorithms, nn: natural neighbours. 4quad: four quadrants criterium, 6near: 6 nearest points, LLF: algorithm suggested in [21], SLS: algorithm suggested in [40]. The first graph shows the rms errors obtained with the single point RBF stencil using inverse multiquadric for 6near and LLF, and Gaussian in the other cases. The second graph presents the density of the sparse matrices of the corresponding linear systems (2). The reference error and density curves for the finite element method (FEM in the legend) are the same as in Figs 3 and 4.

Stencil selection algorithms in Fig. 5.

- Nearest neighbours (6near): $\Xi_{\zeta}$ consists of $\zeta$ and a prescribed number of nearest points in $\Xi \backslash\{\zeta\}$ to $\zeta$, as suggested in one of the first papers on generalised finite difference methods [19]. We choose 6 neighbours to ensure that the density of the matrix $\left[w_{\zeta, \xi}\right]$ is close to the FEM case. The curve in Fig. 5(a) is for $\phi_{\text {imq }}$ as this $\mathrm{RBF}$ performed better than $\phi_{\mathrm{g}}$ and $\phi_{\mathrm{w} 33}$.

- Natural neighbours (nn): $\Xi_{\zeta}$ consists of $\zeta$ and all its natural neighbours, that is the points in $\Xi$ connected to $\zeta$ in the Delaunay triangulation of $\Xi$. To compute the natural neighbours, only a local (albeit sufficiently large to actually include the neighbours) subset of $\Xi$ around $\zeta$ needs to be triangulated. This method for $\mathrm{RBF}$ stencil selection is discussed in $[6,44]$ and used as part of a more complicated algorithm in [35]. Its performance in our tests on FEM centres is very close to that of the 'mesh-dependent' stencil support selection as in Section 4, compare nn curve in Fig. 5(a) with the curves in Fig. 3(a).

- Four quadrants criterium (4quad) goes back to [23] and was used more recently in $[24,3]$. It is designed for the generalised finite difference method, where the stencils are obtained from the local least square fitting by quadratic polynomials on $\Xi_{\zeta}$. Since the number of points in $\Xi_{\zeta} \backslash\{\zeta\}$ is 8 , the matrix density is significantly higher for this method, see Fig. 5(b).

- LLF: algorithm suggested in [21], where first the maximum distance $d_{\zeta}$ from $\zeta$ to 
its four closest neighbours in $\Xi \backslash\{\zeta\}$ is computed, and then $\Xi_{\zeta}$ is defined as the set of all points in $\Xi$ with distance from $\zeta$ not exceeding $\rho d_{\zeta}$ for some constant $\rho>0$. The examples in [21] on non-gridded centres uses $\rho=2.0$. We have chosen $\rho=1.4$ to optimise the rms error in our test. The matrix density for this method is significantly higher than the density of the FEM matrix. We used $\phi_{\text {imq }}$ in this test as suggested in [21].

- SLS: algorithm suggested in [40], which leads to a 6 points set $\Xi_{\zeta}=\left\{\zeta, \xi_{1}, \ldots, \xi_{5}\right\}$ well-posed for bivariate quadratic polynomial interpolation. Since the points $\xi_{i}$ are chosen one after another as the closest to $\zeta$ points in certain subdomains around $\zeta$, this method can be seen as an improvement of the four quadrants criterium. Referring to the notation of [40, Algorithm A], we choose the parameter $\alpha_{0}=0.2$ (in radians) as it is near optimal for our test problem in the sense of the rms error. This is the only method where the matrix $\left[w_{\zeta, \xi}\right]_{\zeta, \xi \in \Xi \backslash \partial \Xi}$ is sparser than the one arising from FEM stencil supports.

Several more specialised (and more complicated) stencil support selection methods have been proposed in the literature, such as Gauss-Jordan pivoting or other techniques to ensure that high order polynomial discrete least square problems are well-conditioned on $\Xi_{\zeta}[38,20,35]$, linear or quadratic programming techniques that seek positive stencils exact for polynomials of certain degree [39], or a search for RBF stencils satisfying a set of conditions that make them especially well suited for certain time dependent PDEs [6].

Note that Algorithm 1 will not perform well if the set $\Xi$ is highly irregular, for example if all 30 closest points to $\zeta \in \Xi \backslash \partial \Xi$ are located in one half-plane going through $\zeta$. This does not happen to the centres of the finite element mesh in our numerical example, and hopefully does not happen to adaptive triangulations generated by appropriate algorithms. More importantly, good methods for the meshless refinement of centres are needed that produce well-behaved discretisation sets $\Xi$, rather than the design of stencil support methods capable to deal with arbitrary irregular centres. The next section presents a first attempt of such a refinement method.

\section{Adaptive meshless refinement}

To describe an adaptive discretisation method, one has to specify an error indicator and a refinement algorithm [2]. We have not addressed the question of a posteriori error estimates for the RBF finite differences because even a priori estimates are not available yet. However, keeping in mind that certain heuristic error indicators are often used successfully for the adaptive mesh refinement (see e.g. [29, 12]), we have chosen for our numerical tests an error indicator based on the comparison of the local variation of the solution $\hat{u}$ of $(2)$.

Error indicator. Given a discretisation of the Dirichlet problem (1), for each $\zeta \in \Xi$ and each $\xi \in \Xi_{\zeta}$ we define the error indicator $\varepsilon(\zeta, \xi)$ associated with the directed edge $\zeta \xi$ by $\varepsilon(\zeta, \xi):=\left|\hat{u}_{\zeta}-\hat{u}_{\xi}\right|$, where $\hat{u}$ is the solution of $(2)$. Let $\bar{\varepsilon}=\bar{\varepsilon}(\Xi):=\max \{\varepsilon(\zeta, \xi)$ : 
$\left.\zeta \in \Xi, \xi \in \Xi_{\zeta}\right\}$. An edge $\zeta \xi$ is marked for refinement if $\varepsilon(\zeta, \xi) \geq \gamma \bar{\varepsilon}$, where $\gamma \in(0,1]$ is a user specified tolerance.

Note that we have decided to assign the error indicator to the edges rather than centres because this seems to us a more universal technique, which may in particular naturally lead to an anisotropic refinement if it is appropriate. Recall that a popular refinement method of triangle bisection in the adaptive finite element method can also be viewed as edge refinement.

We refine an edge $\zeta \xi$ by inserting a new centre in its middle point $(\zeta+\xi) / 2$. However, this point may be located very close to an existing centre $\xi^{\prime} \in \Xi$, or to a new centre already created by the refinement of a different edge. This problem has been recognised in $[3,35]$, where edge refinement algorithms are studied for the generalised finite differences and the finite point method, respectively. Our approach to it is to only proceed with the refinement if the insertion of a new centre does not significantly reduce the local separation in the vicinity of $\zeta$, which we define as

$$
\operatorname{sep}_{\zeta}(\Xi):=\frac{1}{4} \sum_{i=1}^{4} \operatorname{dist}\left(\xi_{i}, \Xi \backslash\left\{\xi_{i}\right\}\right), \quad \zeta \notin \Xi,
$$

where $\xi_{1}, \ldots, \xi_{4}$ are the four closest points in $\Xi$ to $\zeta$, and $\operatorname{dist}(\zeta, \Xi):=\min \left\{\|\zeta-\xi\|_{2}\right.$ : $\xi \in \Xi\}$ is the distance from a point $\zeta$ to a finite set $\Xi$.

For simplicity, we make the following assumptions about the boundary of $\Omega$ and the set $\partial \Xi: 1)$ the connected components of $\partial \Omega$ are simple closed parametric curves, 2) for every simply connected component $\Gamma$ of $\partial \Omega$ the set $\Xi \cap \Gamma$ consists of at least three points, and 3) for each pair of consecutive points $\xi_{1}, \xi_{2} \in \Xi \cap \Gamma$ there is a well defined middle point $\xi^{\prime} \in \Gamma$, e.g. the point corresponding to the average of the parameter values of $\xi_{1}, \xi_{2}$. Then the local refinement of $\partial \Xi$ is well defined, by which we understand the following algorithm: For any $\xi \in \partial \Xi$ find its two neighbours $\xi_{-}, \xi_{+}$in $\partial \Xi$, one on each side of $\xi$ with respect to the parametrisation of the boundary component containing $\xi$, and extend $\partial \Xi$ by inserting the two middle points $\xi_{-}^{\prime}, \xi_{+}^{\prime}$ defined by the pairs $\xi, \xi_{-}$and $\xi, \xi_{+}$.

\section{Algorithm 2. Adaptive meshless refinement}

Input: The set of centres $\Xi$, stencil supports $\left\{\Xi_{\zeta}: \zeta \in \Xi \backslash \partial \Xi\right\}$. Output: The refined set of centres $\Xi^{\prime}$ and stencil supports $\left\{\Xi_{\zeta}^{\prime}: \zeta \in \Xi^{\prime} \backslash \partial \Xi^{\prime}\right\}$. Parameters: $\gamma$ (error indicator tolerance), $\mu$ (separation tolerance). Parameter values used in our numerical experiments: $\gamma=0.5$ for Test Problem 2 and 0.3 for Test Problem $3, \mu=0.7$.

I. Compute the maximum error indicator $\bar{\varepsilon}=\bar{\varepsilon}(\Xi)$ and mark all edges $\zeta \xi, \xi \in \Xi_{\zeta} \backslash\{\zeta\}$, $\zeta \in \Xi \backslash \partial \Xi$, such that $\varepsilon(\zeta, \xi) \geq \gamma \bar{\varepsilon}$. Initialise $\Xi^{\prime}:=\Xi$.

II. For each marked edge $\zeta \xi$ :

1. Let $\xi^{\prime}:=(\zeta+\xi) / 2$.

2. If $\operatorname{dist}\left(\xi^{\prime}, \Xi^{\prime}\right) \geq \mu \operatorname{sep}_{\xi^{\prime}}\left(\Xi^{\prime}\right)$ :

i. Set $\Xi^{\prime}:=\Xi^{\prime} \cup\left\{\xi^{\prime}\right\}$. 
ii. If $\xi \in \partial \Xi$

$$
\text { Set } \Xi^{\prime}:=\Xi^{\prime} \cup\left\{\xi_{-}^{\prime}, \xi_{+}^{\prime}\right\} \text {. }
$$

III. 1. For each $\zeta \in \Xi^{\prime} \backslash \partial \Xi^{\prime}$

i. Apply Algorithm 1 to find $\Xi_{\zeta}^{\prime} \subset \Xi^{\prime}$.

ii. Compute the distances $d_{\xi}=\|\zeta-\xi\|_{2}, \xi \in \Xi_{\zeta}^{\prime} \backslash\{\zeta\}$, and their average $d_{a v}:=\frac{1}{\ell} \sum_{\xi \in \Xi_{\zeta}^{\prime} \backslash\{\zeta\}} d_{\xi}$, where $\ell=\# \Xi_{\zeta}^{\prime}-1$, and mark all edges $\zeta \xi, \xi \in \Xi_{\zeta} \backslash\{\zeta\}$, such that $d_{\xi}>2 d_{a v}$.

2. Repeat Step II for the edges marked in Step III.1 and collect all newly created centres in the set $\Xi_{\text {aux }}$.

3. Apply Algorithm 1 to compute $\Xi_{\zeta}^{\prime}$ for all $\zeta \in \Xi_{\text {aux }}$, and recompute $\Xi_{\zeta}^{\prime}$ for all $\zeta \in \Xi^{\prime} \backslash \Xi_{\text {aux }}$ such that $\operatorname{dist}\left(\zeta, \Xi_{\text {aux }}\right)<d\left(\Xi_{\zeta}^{\prime}\right):=\max _{\xi \in \Xi_{\zeta}^{\prime}}\|\zeta-\xi\|_{2}$.

\section{Remarks}

1. Often $\xi \in \Xi_{\zeta}$ and $\zeta \in \Xi_{\xi}$ simultaneously. In this case the error indicator for the edges $\zeta \xi$ and $\xi \zeta$ has the same value and the potential new centre $(\zeta+\xi) / 2$ is the same. This may be taken into account in Step I by avoiding the edge $\xi \zeta$ after $\zeta \xi$ has been processed.

2. In Step II.2.ii it may happen that $\xi_{-}^{\prime}$ or $\xi_{+}^{\prime}$ is already in $\Xi^{\prime}$ in case that $\xi_{-}$or $\xi_{+}$ belongs to a marked edge, too. To avoid duplication it is sufficient to store the information of which segments of $\partial \Omega$ have been refined.

3. Adjustment of $\Xi^{\prime}$ in Step III helps to improve those stencils that become too irregular with respect to the distances to $\zeta$ after the refinement performed in Step II.

4. If Algorithm 2 does not produce any new centres, it is repeated with a reduced separation tolerance $\mu:=0.9 \mu$ until a minimum number of new centres (10 in our tests) is found.

We have tested Algorithm 2 numerically on Test Problem 2 using as the initial set of centres $\Xi$ the vertices of the triangulation of $\Omega$ obtained by a call of the PDE Toolbox command initmesh with default parameters. The refinements with $\gamma=0.5$ were repeated until a prescribed number of interior centres was generated. Fig. 6 shows typical distributions of the centres obtained in these refinements. Fig. 7 presents the errors of the RBF methods as well as a matrix density curve. The numerical results in Fig. 7 are similar to those in Fig. 4, but in contrast to them the discretisation centres are now generated by a purely meshless method.

Finally, we apply the same method to a different test problem.

Test Problem 3. [3] Dirichlet problem (1) for the Laplace equation $\Delta u=0$ in the domain $\Omega=(0.01,1.01)^{2}$ with boundary conditions chosen such that the exact solution is $u(x, y)=\log \left(x^{2}+y^{2}\right)$. 

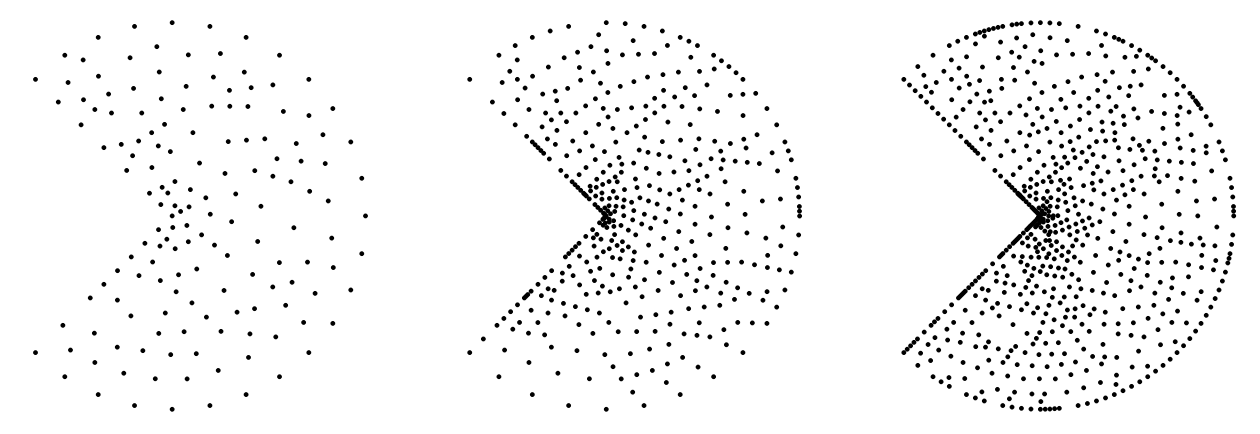

Figure 6: Typical adaptive meshless discretisations of $\Omega$ generated by Algorithm 2 for Test Problem 2

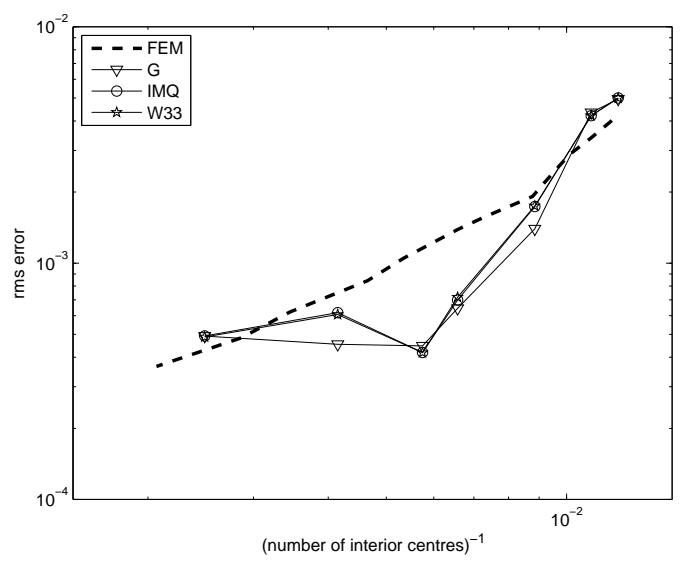

(a) Single point stencils

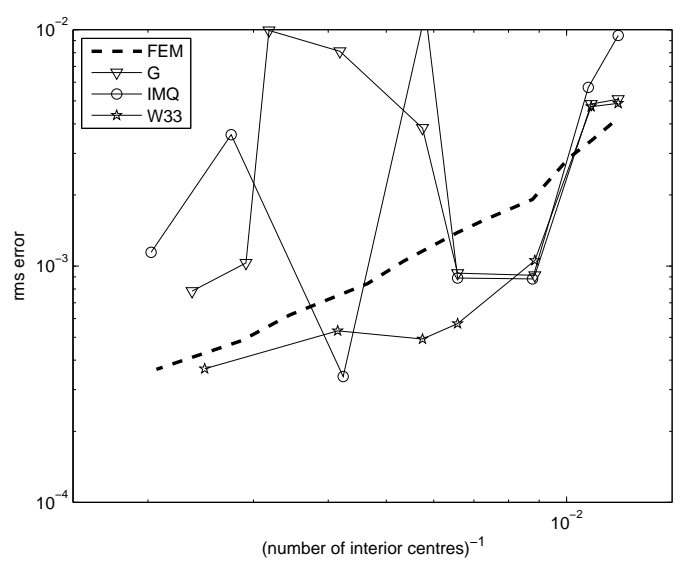

(c) Hermite stencils

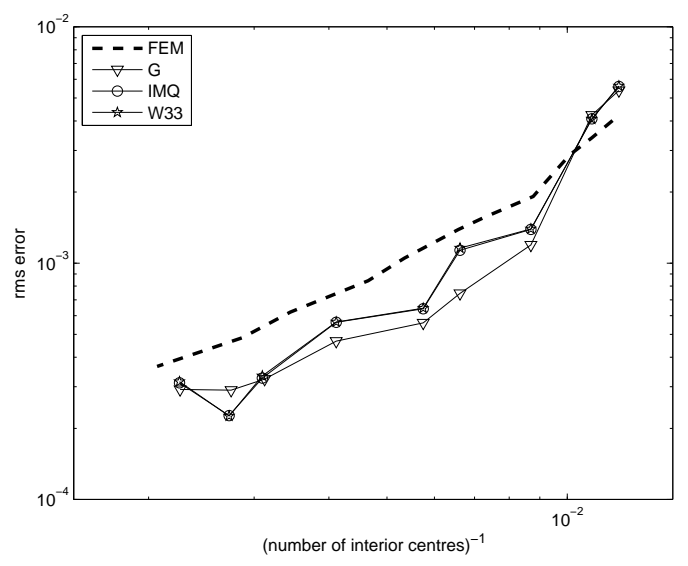

(b) Multipoint stencils

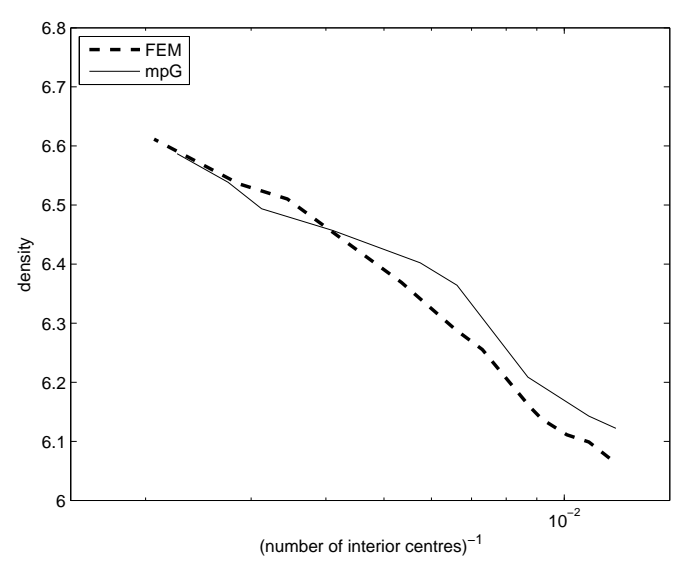

(d) Matrix density

Figure 7: RBF solution on adaptive meshless centres generated according to Algorithms 1 and 2 for Test Problem 2. The first three graphs show the error obtained with three types of RBF stencils, whereas the forth graph presents the density of the sparse matrix of the linear system (2) for both FEM and multipoint Gaussian RBF stencil methods. 
Numerical results presented in Fig. 8 are obtained by applying Algorithm 2 to Test Problem 3, where we used the tolerance value $\gamma=0.3$. We can see that the RBF errors (except of Hermite stencils with $\phi_{\mathrm{g}}$ and $\phi_{\mathrm{imq}}$ ) compare well to the error of the finite element method, sometimes being up to 4 times smaller, whereas the density of the matrix of (2) remains close to the density of the FEM stiffness matrix.

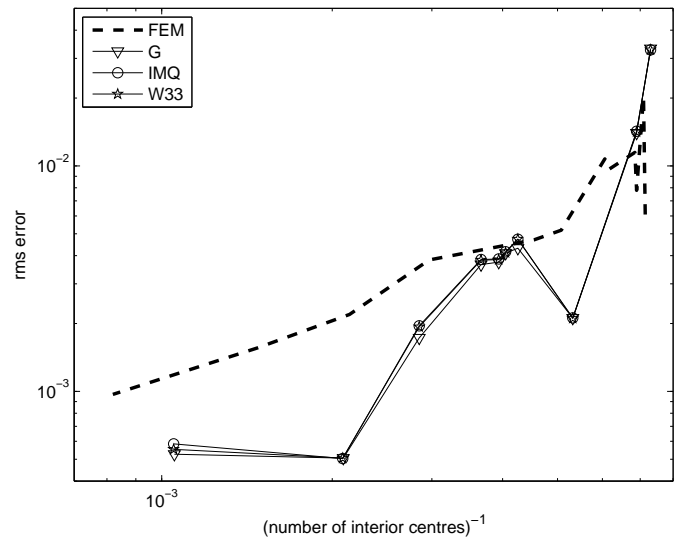

(a) Single point stencils

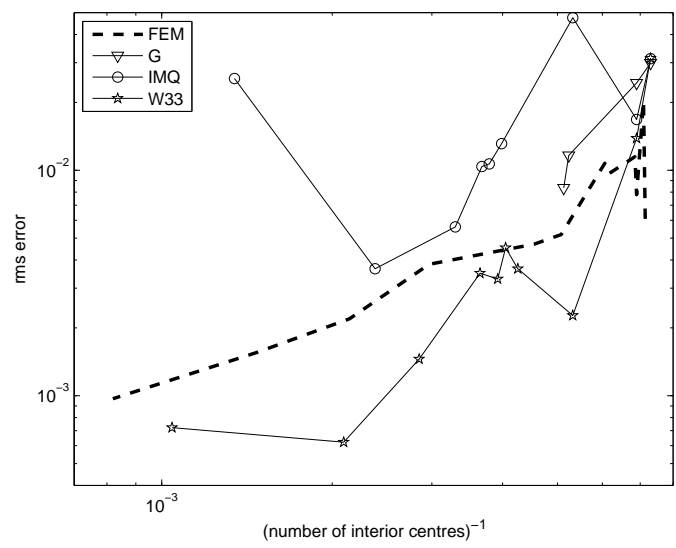

(c) Hermite stencils

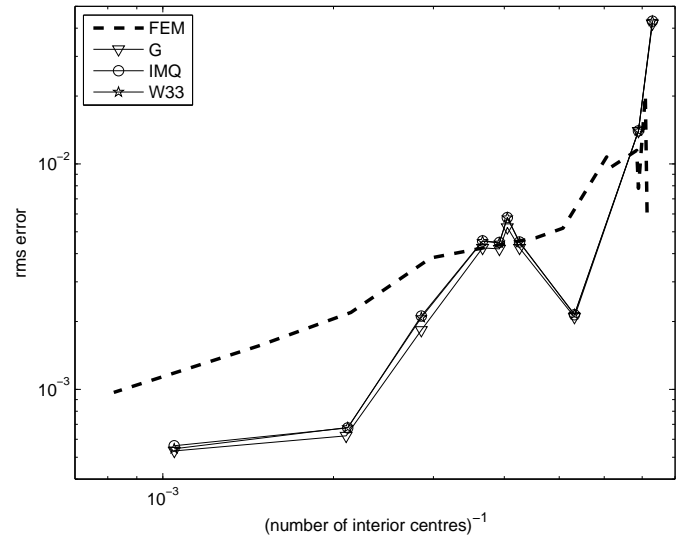

(b) Multipoint stencils

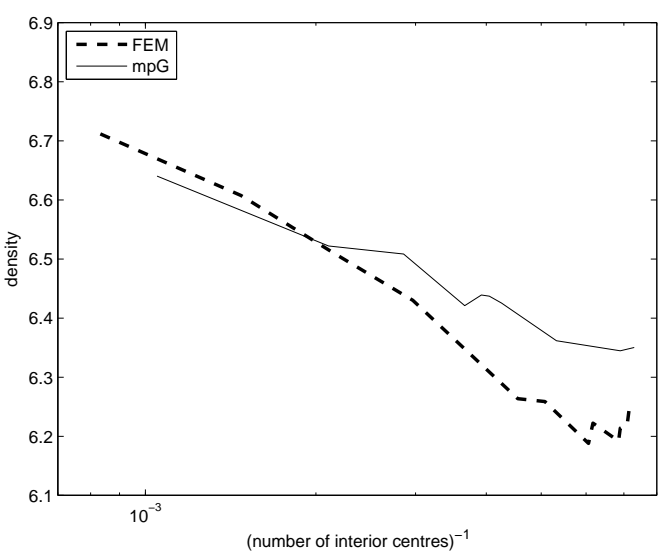

(d) Matrix density

Figure 8: RBF solution on adaptive meshless centres generated according to Algorithms 1 and 2 for Test Problem 3. The first three graphs show the error obtained with three types of RBF stencils, whereas the forth graph presents the density of the sparse matrix of the linear system (2) for both FEM and multipoint Gaussian RBF stencil methods.

\section{Conclusion and future work}

The main outcome of the work presented in this paper is that numerical differentiation stencils arising from RBF interpolation can be used to generate adaptive meshless discretisations of the Poisson equation in $2 \mathrm{D}$ capable to compete with the adaptive finite element method by providing solutions of comparable, if not better, accuracy, while maintaining approximately the same density of the linear systems to be solved. 
We hope that these results will help to develop effective meshless methods for the more complex problems where mesh generation is challenging. An obvious route of future research is to investigate higher order methods, where larger stencils are selected, and other types of PDE problems. Algorithms 1 and 2 need to be further investigated with a goal to develop them to the point where rigorous statements about the guaranteed quality of the centres and stencils can be made similar to what is known for the adaptive mesh generation methods, where it is known for example that the bisection algorithm on triangular meshes always produces shape regular triangles [31]. The selection of the scaling parameter $\delta$ needs improvement as our current approach seems to be restricted to relatively coarse discretisations. In particular, the application of ContourPadé techniques of [44] may lead to a significant improvement of the error of the RBF based meshless methods. Theoretical understanding of these methods, in particular $a$ priori and a posteriori error bounds, is a very interesting topic that requires nontrivial new ideas.

\section{References}

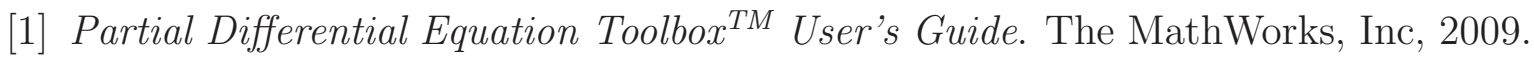

[2] I. Babuska and T. Strouboulis. The Finite Element Method and its Reliability. Oxford University Press, London, 2001.

[3] J. Benito, F. Ureña, L. Gavete, and R. Alvarez. An h-adaptive method in the generalized finite differences. Comput. Methods Appl. Mech. Eng., 192(5-6):735$759,2003$.

[4] J. P. Boyd and L. Wang. Truncated Gaussian RBF differences are always inferior to finite differences of the same stencil width. Commun. Comput. Phys., 5:42-60, 2009 .

[5] M. D. Buhmann. Radial Basis Functions. Cambridge University Press, New York, NY, USA, 2003.

[6] T. Cecil, J. Qian, and S. Osher. Numerical methods for high dimensional hamiltonjacobi equations using radial basis functions. J. Comput. Phys., 196(1):327-347, 2004 .

[7] O. Davydov. On the approximation power of local least squares polynomials. In J. Levesley, I. J. Anderson, and J. C. Mason, editors, Algorithms for Approximation $I V$, pages 346-353. University of Huddersfield, UK, 2002.

[8] O. Davydov. Error bound for radial basis interpolation in terms of a growth function. In A. Cohen, J.-L. Merrien, and L. L. Schumaker, editors, Curve and Surface Fitting: Avignon 2006, pages 121-130. Nashboro Press, Brentwood, 2007.

[9] O. Davydov, R. Morandi, and A. Sestini. Local hybrid approximation for scattered data fitting with bivariate splines. Computer Aided Geometric Design, 23(9):703$721,2006$. 
[10] O. Davydov, A. Sestini, and R. Morandi. Local RBF approximation for scattered data fitting with bivariate splines. In M. G. de Bruin, D. H. Mache, and J.Szabados, editors, Trends and Applications in Constructive Approximation. ISNM Vol.151, pages 91-102. Birkhäuser, 2005.

[11] O. Davydov and F. Zeilfelder. Scattered data fitting by direct extension of local polynomials to bivariate splines. Adv. Comput. Math., 21(3-4):223-271, 2004.

[12] H. Ding and C. Shu. A stencil adaptive algorithm for finite difference solution of incompressible viscous flows. J. Comput. Phys., 214(1):397-420, 2006.

[13] Q. Du, M. Gunzburger, and L.-L. Ju. Meshfree, probabilistic determination of point sets and support regions for meshless computing. Comput. Meths. Appl. Mech. Engrg., 191:1349-1366, 2002.

[14] C. Duarte and J. Oden. An h-p adaptive method using clouds. Methods Appl. Mech. Eng., 139(1-4):237-262, 1996.

[15] G. F. Fasshauer. Meshfree Approximation Methods with MATLAB. World Scientific Publishing Co., Inc., River Edge, NJ, USA, 2007.

[16] M. S. Floater and A. Iske. Multistep scattered data interpolation using compactly supported radial basis functions. J. Comput. Appl. Math., 73(1-2):65-78, 1996.

[17] S. R. Idelsohn and E. Oñate. To mesh or not to mesh. That is the question... Comput. Meths. Appl. Mech. Engrg., 195:4681-4696, 2006.

[18] A. Iske and T. Sonar. On the structure of function spaces in optimal recovery of point functionals for ENO-schemes by radial basis functions. Numer. Math., 74(2):177-201, 1996.

[19] P. S. Jensen. Finite difference techniques for variable grids. Comput. Struct., 2(12):17-29, 1972.

[20] M. Kamruzzaman, T. Sonar, T. Lutz, and E. Krämer. A new meshless collocation method for partial differential equations. Commun. Numer. Meth. Eng., 24(12):1617-1639, 2008.

[21] C. K. Lee, X. Liu, and S. C. Fan. Local multiquadric approximation for solving boundary value problems. Comput. Mech., 30(5-6):396-409, 2003.

[22] X.-Y. Li, S.-H. Teng, and A. Üngör. Point placement for meshless methods using sphere packing and advancing front methods. In ICES00: International Conference on Computational Engineering Science, Los Angeles, USA, 20-25 August 2000.

[23] T. Liszka and J. Orkisz. The finite difference method at arbitrary irregular grids and its application in applied mechanics. Comput Struct, 11:83-95, 1980.

[24] T. J. Liszka, C. A. M. Duarte, and W. W. Tworzydlo. hp-meshless cloud method. Comp. Meth. Appl. Mech. Engrg., 139:263-288, 1996. 
[25] G. R. Liu and Y. T. Gu. A local radial point interpolation method (LR-PIM) for free vibration analyses of 2-D solids. J. Sound Vibr., 246(1):29-46, 2001.

[26] Y. Liu, Y. C. Hon, and K. M. Liew. A meshfree Hermite-type radial point interpolation method for Kirchhoff plate problems. Int. J. Numer. Meth. Eng., 66:1153-1178, 2006.

[27] H. Lu and J. Chen. Adaptive Galerkin particle method. Lect. Notes Comput. Sci. Eng., 26:251-267, 2002.

[28] R. Löhner and E. Oñate. An advancing point grid generation technique. Commun Numer Methods Eng., 14(12):1097-1108, 1998.

[29] D. F. Martin and K. L. Cartwright. Solving Poisson's equation using adaptive mesh refinement. Technical Report UCB/ERI M96/66, UC Berkeley, 1996.

[30] J. M. Melenk. On approximation in meshless methods. In J. Blowey and A. Craig, editors, Frontiers in Numerical Analysis Durham 2004, pages 65-141. Springer Verlag, 2005.

[31] W. F. Mitchell. A comparison of adaptive refinement techniques for elliptic problems. ACM Transactions on Mathematical Software, 15(4):326-347, 1989.

[32] H. Netuzhylov. A Space-Time Meshfree Collocation Method for Coupled Problems on Irregularly-Shaped Domains. PhD thesis, TU Braunschweig, CSE - Computational Sciences in Engineering, 2008.

[33] V. P. Nguyen, T. Rabczuk, S. Bordas, and M. Duflot. Meshless methods: A review and computer implementation aspects. Math. Comput. Simul., 79(3):763-813, 2008.

[34] E. Oñate, S. Idelsohn, O. C. Zienkiewicz, R. L. Taylor, and C. Sacco. A stabilized finite point method for analysis of fluid mechanics problems. Comp. Methods Appl. Mech. Eng., 39:315-346, 1996.

[35] F. Perazzo, R. Löhner, and L. Perez-Pozo. Adaptive methodology for meshless finite point method. Adv. Eng. Softw., 39(3):156-166, 2008.

[36] T. Rabczuk and T. Belytschko. Adaptivity for structured meshfree particle methods in 2D and 3D. International Journal for Numerical Methods in Engineering, 63(11):1559-1582, 2005.

[37] R. Schaback. Error estimates and condition numbers for radial basis function interpolation. Adv. Comput. Math, 3:251-264, 1995.

[38] W. Schönauer and T. Adolph. How WE solve PDEs. J. Comput. Appl. Math., 131(1-2):473-492, 2001.

[39] B. Seibold. Minimal positive stencils in meshfree finite difference methods for the Poisson equation. Comput. Methods Appl. Mech. Eng., 198(3-4):592-601, 2008. 
[40] L. Shen, G. Lv, and Z. Shen. A finite point method based on directional differences. SIAM Journal on Numerical. Analysis, 47(3):2224-2242, 2009.

[41] C. Shu, H. Ding, and K. S. Yeo. Local radial basis function-based differential quadrature method and its application to solve two-dimensional incompressible Navier-Stokes equations. Comput. Methods Appl. Mech. Eng., 192(7-8):941-954, 2003.

[42] A. I. Tolstykh and D. A. Shirobokov. On using radial basis functions in a 'finite difference mode' with applications to elasticity problems. Computational Mechanics, 33(1):68-79, 2003.

[43] H. Wendland. Scattered Data Approximation. Cambridge University Press, 2005.

[44] G. B. Wright and B. Fornberg. Scattered node compact finite difference-type formulas generated from radial basis functions. J. Comput. Phys., 212(1):99-123, 2006. 